Ekbom, K., Lindholm, H. \& Ljungbers, I. (1972) New dystonic syndrome associated with butyrophenone therapy. Zeitschrift für Neurologie, 202. 94-103.

Kurtz, G., Kapihammer, H. P. \& Peuker, B. (1993) Pisa syndrome in clozapine therapy. Nervenorzt, 64 . 742-746.

Zimbroff, D. L., Kane, J. M., Tammiga, C. A., et of (1997) Controlled, dose-response study of sertindole and haloperidol in the treatment of schizophrenia. Sertindole Study Group. American journal of Psychiatry. I54, 782-79I.

F. Padberg, S. Stübner, K. Buch, U. Hegerl, H. Hampel Department of Psychiatry, Geriatric Psychiatry Branch, Lugwig-Maximilian University, School of Medicine, Nussbaumstr. 7, 80336 Munich, Germany

\section{Olanzapine-induced thrombocytopenia in association with idiopathic thrombocytopenic purpura}

Sir: Thombocytopenia is one of the common side-effects of pharmacological therapy, but it is rarely induced by psychotropic drugs. We would like to draw attention to a case where the conditions of both an idiopathic and a neuroleptic-induced thrombocytopenia occurred.

A 30-year-old woman had a four-year history of paranoid schizophrenia. She was hospitalised because of a grand-mal seizure and persistent delusions while receiving clozapine. She presented with psychomotor retardation, affective blunting and delusions of reference. Routine examinations were unremarkable, except a platelet count of $137 / \mathrm{nl}$. Medical records on previous hospitalisations elsewhere report low platelet counts of $107 / \mathrm{nl}$ and $117 / \mathrm{nl}$ prior to receiving medication; and she had a history of petechia. However, 11 years ago platelets had been within normal range. She made a good recovery on benperidol. To meet psychomotor side-effects, we then prescribed olanzapine $(20 \mathrm{mg})$. While checking laboratory values regularly we discovered a decrease in platelets to $10 / \mathrm{nl}$ on the 17th day of treatment with olanzapine. This decrease had occurred in three days. The patient was transferred to a general medical ward. Platelets returned to normal after discontinuation of olanzapine and administration of human gamma globulins and prednisolone. The patient was discharged on $\mathbf{3 0 0} \mathrm{mg}$ sulpiride and a maintenance dose of prednisolone.

We assumed that olanzapine had worsened a pre-existing idiopathic thrombocytopenic purpura (ITP) on an autoimmune basis. To confirm this diagnosis, we repeatedly attempted to document the presence of autoantibodies directed against the complex formed by the drug binding to the thrombocyte membrane. This is possible in about $40 \%$ of all presumed cases of druginduced thrombocytopenia (Greinacher et $a l, 1994)$. Since we failed, the attribution of thrombocytopenia to autoimmune drugdependent destruction was not proved definitively, and relies on the time-dependence of the abnormal blood count on the administration of olanzapine. Thrombocytopenia usually occurs 5-15 days after starting a drug therapy (Handin, 1998).

Previous reports support the hypothesis that psychiatric medication can worsen the condition of a pre-existing ITP (König et al, 1995) or induce an immune-mediated thrombocytopenia (Balon et al, 1987; Durst et al, 1993; Mahmood et al, 1996). Therefore, this case raises the issue of a coincidence or a possible interdependence between an idiopathic tendency to thrombocytopenia as in ITP, and drug effects of olanzapine.

Babon, R., Berchou, R. \& Zethelius, M. (1987) Thrombocytopenia associated with chlorpromazine. haloperidol and thiothixene: a case report. Conodion journal of Psychiotry, 32, 49-50.

Durst, R., Dorevitch, A. \& Fraenkel, Y. (1993) Platelet dysfunction association with clozapine therapy. Southern Medical Journal, 86, 1170-1172.

Greinacher, A., Pützsch, B., Amiral, J., et al (1994) Heparin-associated thrombocytopenia: isolation of the antibody and characterization of a multimolecular PF4 heparin complex as the major antigen. Thrombosis and Hoemostosis, 71, 247-25I.

Handin, R. I. (1998) Clotting disorders. In Harrison's Principles and Practice of Internal Medicine (14th edn) (eds K. J. Isselbacher, E. Braunwald, J. D. Wilson et al), pp. 730-736. New York: McGraw Hill.

König, F., Stumpp, W., Wolfersdorf, M., et al (1995) Verlauf eines Morbus Werthof nach Therapiebeginn mit Maprotilin. Nervenorzt, 66, 60-65.

Mahmood, T., Silverstone, T. \& Spittle, B. (1996) Risperidone appears safe in patients with antipsychoticinduced blood dyscrasias. International Clinical Psychophormacology. II, 53-54.

\section{S. Bachmann, J. Schröder, J. Pantel,}

C. Mundt Department of Psychiatry, University of Heidelberg, Voßstr. 4, 69115 Heidelberg, Germany

M. Zorn, M. Witzens, G. Egerer Department of Internal Medicine and Medical Policlinic V.

University of Heidelberg, Bergheimer Str. 52 and

Hospitalstr. 3, 69115 Heidelberg, Germany

\section{Salinophagia in anorexia nervosa}

Sir: We report a case of pathological salt ingestion as a feature of anorexia nervosa.
The patient is a single woman in her thirties with a 15-year history of anorexia nervosa (World Health Organization, 1992), of sufficient severity to necessitate in-patient treatment on a specialist unit. While engaged in our standardised treatment programme, combining weight gain with psychotherapy, she admitted to intermittent pathological ingestion of table salt over the preceding two years in the form of up to 20 packets (approximately $80 \mathrm{~g}$ ) of salt per day, which she would consume with bread or potatoes. Her impulses towards salt ingestion existed in negative reciprocity with her body mass index, and came to light as her weight reached the mean matched population weight. Despite this history her electrolyte levels were normal, with adequate renal compensation of hypernatraemia.

The phenomenology of her behaviour appeared to be a form of deliberate selfharm, ego-syntonic but self-punative in nature. In particular, her salt ingestion lacked salient features of an obsessivecompulsive disorder or pica. Notably, another patient on the unit appeared to adopt similar behaviour in imitation, which reflects the tendency for some symptoms of anorexia nervosa to run in trends.

We addressed her salt ingestion as a form of learned maladaptive behaviour, combining both cognitive-behavioural and psychodynamic techniques, and the patient remains in treatment.

Compulsive eating of unusual substances has been described in a variety of psychiatric disorders, including schizophrenia, learning disability (Jawed et al, 1993) and anorexia nervosa (McLoughlin \& Hassanyeh, 1990). The latter description linked pagophagia (the compulsive eating of ice) with iron and zinc deficiency. However, our patient's behaviour was not compulsive in nature and, to our knowledge, is the first published description of pathological salt ingestion, or 'salinophagia', as a symptom of anorexia nervosa. Although rare, we feel it should be added to the list of maladaptive behaviours associated with anorexia nervosa and bulimia nervosa. In addition, physicians should consider salinophagia among their differential diagnoses when faced with unexplained compensated or uncompensated hypernatraemia.

Jamed, S. H., Krishnan, V. H., Prasher, V. P., of ol (1993) Worsening pica as a symptom of depressive illness in a person with severe mental handicap. British journal of Psychiatry, 162, 835-837. 
McLoughlin, I. J. \&assamyeh, F. (1990) Pica in a patient with anorexia nervosa. British journal of Psychiotry, 156, 568-570.

World Health Organization (1992) Tenth Revision of the International Classification of Diseases (ICD-10). Geneva: WHO.

J. F. Morgan, J. H. Lacey Division of General Psychiatry, Department of Mental Health Sciences, St George's Hospital Medical School, London SWI7 ORE

\section{Fluoxetine and graded exercise in chronic fatigue syndrome}

Sir: First, may we congratulate Wearden $\boldsymbol{e t}$ al (1998) on completing such an important and technically difficult study of chronic fatigue syndrome (CFS). We wondered whether the authors were able to give further data which might explain the relatively modest results with fluoxetine and which are also relevant to the commentary on this study (Deale et al, 1998).

Many patients in such studies may have long illness durations and in this case the median duration of fatigue symptoms for all patients was over two years. It was unclear how long the duration of mood symptoms was, bearing in mind that major depression and dysthymia were important components of the psychiatric comorbidity in $46 \%$ of patients.

These issues are important as, in our experience at a multi-disciplinary CFS clinic, most patients will have been offered a range of antidepressants before referral to tertiary care and it is possible that these mood symptoms may have become refractory to treatment (Scott, 1988). This might partly explain the very modest response to fluoxetine seen and is an alternative explanation to that suggested by Deale et al (1998). The neuroendocrine hypothesis of CFS is indeed of great interest but not all of these findings have been consistently replicated (Yatham et al, 1995), which may point to heterogeneity in this patient group.

\section{Deale, A., Chalder, T. \& Wesseby, S. (1998)}

Commentary on: Randomised, double-blind, placebocontrolled trial of fluoxetine and graded exercise for chronic fatigue syndrome. British journal of Psychiatry. 172. 491-492.

Scott, J. (1988) Chronic depression. British Journal of Psychiatry, 153, 287-297.

Wearden, A. I., Morriss, R. K., Mullis, R., et of (1998) Randomised, double-blind, placebo-controlled treatment trial of fluoxetine and graded exercise for chronic fatigue syndrome. British journal of Psychiatry. I72, 485-490.
Yatham, L. N., Morehouse, R. L., Chisholm, B.T., et al (1995) Neuroendocrine assessment of serotonin (5-HT) function in chronic fatigue syndrome. Canadian journal of Psychiatry, 40, 93-96.

S. Lynch, J. Fraser Division of Psychiatry and Behavioural Sciences in Relation to Medicine, Clinical Sciences Building, St James's University Hospital, Beckett Street, Leeds LS9 7TF

Author's reply: Drs Lynch and Fraser raise an important question concerning the duration of symptoms of depression in our trial of fluoxetine in patients with CFS (Wearden et al, 1998) and in other CFS trials. Unfortunately, we do not have data on the duration of depressive symptoms, although symptoms of depressive or anxiety disorder preceded symptoms of fatigue in only nine $(7 \%)$ of the 136 patients with CFS. In our paper we showed that patients with depressive disorders at baseline randomly allocated to fluoxetine or placebo tended to improve over the six-month period, which does not support the assertion that there was a substantial proportion of patients in our sample with difficult-totreat chronic depression.

Possibly one reason why fluoxetine was shown overall to have a modest effect over six months is because there was a differential effect of fluoxetine on CFS patients with or without any depressive diagnosis at baseline. The 26 patients with any DSM-III-R depressive disorder randomly allocated to fluoxetine showed a mean improvement between baseline and six months of 3.2 (95\% CI 1.6-4.8) on the Hospital Anxiety and Depression (HAD) scale depression score and $3.5 \mathrm{mlO}_{2} / \mathrm{kg}$ per $\min (95 \%$ CI 1.3-5.7) on the Functional Work Capacity (FWC) measure. The 20 patients with any DSM-III-R depressive disorder randomly allocated to placebo showed a mean improvement over the same time scale on the HAD depression scale of 1.7 (95\% CI 0-3.3) and FWC measure of $0.3 \mathrm{mlO}_{2} / \mathrm{kg}$ per min $(95 \% \mathrm{CI}$ -1.9-2.4). The 42 patients without DSMIII-R depressive disorder randomly allocated to fluoxetine showed a mean improvement of 0.9 (95\% CI $-0.1-1.9)$ on the HAD depression scale and $0.2 \mathrm{mlO}_{2} / \mathrm{kg}$ per $\min (95 \% \mathrm{CI}-1.1-1.5)$ on the FWC measure. The 48 patients without DSMIII-R depressive disorder randomly allocated to placebo showed a mean improvement of 0.9 (95\% CI $0.04-1.9)$ on the $\mathrm{HAD}$ depression scale and $1.4 \mathrm{mlO}_{2} / \mathrm{kg}$ per $\min (95 \% \mathrm{CI}-0.1-3.0)$ on the FWC measure. Although this is a post-hoc analysis which must be treated with great caution, it suggests that fluoxetine has a modest effect on depression and functional work capacity in depressed CFS patients only. Fluoxetine provides no benefit over placebo in the treatment of CFS patients with no depression.

As we suggested in the discussion section of our paper, the overall effect of fluoxetine on depression in the whole sample is dependent on the proportion of patients in the sample with a diagnosis of depression.

Wearden A. J., Morriss, R. K., Mullis, R., et al (1998) Randomised, double-blind, placebo-controlled treatment trial of fluoxetine and graded exercise for chronic fatigue syndrome. British journal of Psychiotry. I72, $485-490$

R. Morriss Guild NHS Trust, Royal Preston Hospital, Sharoe Green Lane, Preston PR2 9HT

\section{Chromosome 22qll deletions and aggressive behaviour}

Sir: We thank Lachman \& Papolos (1998) for their interest in our paper on the prevalence of velo-cardio-facial syndrome (VCFS) in a population of subjects with idiopathic learning disability (Murphy et al, 1998) and agree that aggressive behaviour was a feature of the clinical presentation of both patients reported. Lachman \& Papolos (1998) suggest that, as the low-activity catechol-O-methyltransferase (COMT) allele is associated with violence in schizophrenia, hemizygosity for this allele may be the common denominator that leads to aggression in individuals with VCFS and schizophrenia.

To test this hypothesis, we genotyped the COMT codon 158 polymorphism in both individuals with VCFS and schizophrenia reported in our study (Murphy et al, 1998). Both individuals were found to be hemizygous for the low-activity COMT allele. Consequently, our results lend support to the hypothesis proposed by Lachman \& Papolos (1998) that hemizygosity for the low-activity COMT allele may be a determinant for aggressive behaviour in individuals with schizophrenia with or without VCFS.

Lachman, H. M. \& Papolos, D. F. (1998) Chromosome 22qll deletions and aggressive behaviour (letter). British journal of Psychiatry, 172, 540.

Murphy, K. C., Jones, R. G., Griffiths, E., et al (1998) Chromosome 22qll deletions. An under-recognised 